\title{
Comparison of Synovial Fluid and Serum Procalcitonin for Diagnosis of Periprosthetic Joint Infection: A Pilot Study in 32 Patients
}

\begin{abstract}
Paphon Sa-ngasoongsong $(\mathbb{D}$, Siwadol Wongsak $(\mathbb{D})$, Chavarat Jarungvittayakon, Kawee Limsamutpetch, Thanaphot Channoom, and Viroj Kawinwonggowit
\end{abstract}

Department of Orthopedics, Faculty of Medicine Ramathibodi Hospital, Mahidol University, Bangkok, Thailand

Correspondence should be addressed to Siwadol Wongsak; siwadolrama@hotmail.com

Received 30 March 2018; Revised 16 September 2018; Accepted 17 September 2018; Published 1 October 2018

Guest Editor: Berardo Di Matteo

Copyright (C) 2018 Paphon Sa-ngasoongsong et al. This is an open access article distributed under the Creative Commons Attribution License, which permits unrestricted use, distribution, and reproduction in any medium, provided the original work is properly cited.

\begin{abstract}
Background. Periprosthetic joint infection (PJI) remains challenging since a "gold standard" for diagnosis has not yet been established. This study aimed to evaluate the accuracy of synovial fluid procalcitonin (SF-PCT) and serum procalcitonin as a diagnostic biomarker for PJI and to compare its accuracy against standard methods. Methods. A prospective cohort study was conducted during 2015-2017 in 32 patients with painful hip or knee arthroplasty who have underwent revision surgery. Relevant clinical and laboratory data were collected. PJI was diagnosed based on the 2013 international consensus criteria. Preoperative blood sample and intraoperatively acquired joint fluid were taken for PCT measurement with a standard assay. Diagnostic accuracy was analyzed by the receiver-operating characteristic curve and the area under the curve (AUC). Results. Twenty patients (62.5\%) were classified as the PJI group, and $12(37.5 \%)$ were classified as the aseptic loosening group. The median age was 68 years (range 38-87 years). The median values of SF-PCT and serum PCT in the PJI group were both significantly higher than those in the aseptic loosening group: the median serum PCT levels (interquartile range: IQR) were $0.33 \mathrm{ng} / \mathrm{mL}(0.08-2.79 \mathrm{ng} / \mathrm{mL})$ in the PJI group compared with $0.04 \mathrm{ng} / \mathrm{mL}(0.03-0.06 \mathrm{ng} / \mathrm{mL})$, and the median SF-PCT levels (IQR) were $0.16 \mathrm{ng} / \mathrm{mL}(0.12-0.26 \mathrm{ng} / \mathrm{mL}) \mathrm{in}$ PJI group compared with $0.00(0.00-0.00 \mathrm{ng} / \mathrm{mL})(p<0.001$, both). SF-PCT, with a cut-off level of $0.08 \mathrm{ng} / \mathrm{mL}$, had an AUC of 0.87 , a sensitivity of $90.0 \%$, a specificity of $83.3 \%$, and a negative likelihood ratio (LR-) of 0.12 . Serum PCT, with a standard cut-off level of $0.5 \mathrm{ng} / \mathrm{mL}$, had an AUC of 0.70 , a sensitivity of $40.0 \%$, a specificity of $100.0 \%$, and a LR- of 0.60 . Conclusion. SF-PCT appears to be a reliable test and could be useful as an alternative indicator or in combination with standard methods for diagnosing PJI.
\end{abstract}

\section{Introduction}

Periprosthetic joint infection (PJI) is a serious complication after total joint arthroplasty resulting in devastating consequences, such as revision surgery, limb loss, or death [1-3]. However, the diagnosis of this condition is difficult and often delayed, especially with chronic or low-grade infections, due to the lack of "gold standard" examinations and limited accuracy with current diagnostic methods. Therefore, a combination of preoperative and intraoperative markers-including synovial fluid cell count/differential, serum inflammatory markers, cultures, clinical signs, and tissue pathology-are required for PJI diagnosis. Recent studies regarding the new diagnostic techniques demonstrated that some biomarkers-such as procalcitonin (PCT), interleukin6 (IL-6), and $\alpha$-defensin-are helpful and a better marker for PJI [4-6]. Moreover, several studies also showed that the synovial fluid biomarkers obtained directly from the infected joint are more reliable and accurate for diagnosing PJI compared to serum biomarkers and other existing tests $[7,8]$.

PCT, the precursor of calcitonin, is a 116-amino-acid protein produced by the neuroendocrine and the parafollicular cells of the thyroid. Serum PCT level is generally very low $(<0.05 \mathrm{ng} / \mathrm{mL})$ in healthy subjects [9], but specifically elevates in bacterial and fungal infections [10]. It is also unresponsive or only mildly reactive to aseptic inflammation and viral infection [11]. Therefore, numerous studies have 
TABLE 1: Definition for periprosthetic joint infection by International Consensus Group.

Periprosthetic joint infection (PJI) is present when one major criterion exists or when three of five minor criteria exist

Major criteria $\quad$ (1) Two positive periprosthetic cultures with phenotypically identical organisms

(2) A sinus tract communicating with the joint

Minor criteria

(1) Elevated serum C-reactive protein AND erythrocyte sedimentation rate

(2) Elevated synovial fluid white blood cell (WBC) count OR ++change on leukocyte esterase test strip

(3) Elevated synovial fluid polymorphonuclear neutrophil percentage

(4) Positive histological analysis of periprosthetic tissue

(5) A single positive culture

TABLE 2: The threshold for the minor diagnostic criteria according to the International Consensus Group.

\begin{tabular}{lcc}
\hline Criterion & Acute PJI (<90 days) & Chronic PJI (>90 days) \\
\hline Erythrocyte sedimentation rate $(\mathbf{m m} / \mathbf{h r})$ & Not helpful; no threshold was determined & 30 \\
C-reactive protein $(\mathbf{m g} / \mathbf{L})$ & 100 & 10 \\
Synovia white blood cell count $($ cells/ $\boldsymbol{\mu L})$ & 10,000 & 3,000 \\
Synovial polymorphonuclear percentage $\mathbf{( \% )}$ & 90 & 80 \\
Leukocyte esterase & + or ++ & Same as acute \\
Histological analysis of tissue & $>5$ neutrophils/HPF in 5 HPFs $(\mathrm{x} 400)$ & Same as acute \\
\hline
\end{tabular}

PJI: periprosthetic joint infection; HPF: high power field.

shown its ability for differentiating septic arthritis from the aseptic condition [12-14]. Regarding the accuracy with PJI diagnosis, a recent meta-analysis showed that serum PCT had a pooled sensitivity and a pooled specificity for detecting PJI as $53 \%$ and $92 \%$, respectively [15]. However, to the best of our knowledge, while serum PCT seems reliable $[4,15,16]$, only a few studies addressed the efficacy of synovial fluid PCT (SFPCT) for PJI diagnosis [13], and its diagnostic utility has not been clearly established. The aim of this study was to assess synovial fluid and serum levels of PCT as a diagnostic tool for PJI and to evaluate their diagnostic accuracy compared with the standard tests.

\section{Patients and Methods}

2.1. Study Design, Inclusion, and Exclusion Criteria. This study design was a single-centered prospective cohort study in a medical university hospital, and the study was approved by the institutional board review committee (Faculty of Medicine Ramathibodi Hospital, Mahidol University: Protocol number ID 05-58-01). All patients signed informed consent forms prior to being enrolled. The study was conducted in accordance with the declaration of Helsinki. Between 2015 and 2017, patients undergoing revision hip or knee arthroplasty were recruited into this prospective study. The patient-inclusion criteria were (1) pain at the site of total hip or total knee arthroplasty that prompted a clinical evaluation for infection or possible revision hip or knee arthroplasty, (2) no history of previous septic arthritis treatment or previous septic revision surgery, (3) sufficient synovial fluid for the study methods, and (4) sufficient clinical and laboratory data for PJI classification according to the criteria of the International Consensus Meeting on Periprosthetic Joint Infection 2013 [17] (Tables 1 and 2). Patients were excluded if they received any antibiotics or joint puncture treatments prior to enrollment in the current study.

All patients underwent standard diagnostic evaluation for PJI diagnosis. Preoperative blood samples were taken for complete blood count (CBC) erythrocyte sedimentation rate (ESR), c-reactive protein (CRP), and PCT. Joint aspiration was done intraoperatively before opening the joint capsule, and then synovial fluid was sent for cell differentiation, cell count, gram stain, aerobic culture, and PCT. Intraoperative frozen section was performed. Periprosthetic tissue from five different areas (joint capsule, synovial lining, intramedullary material, granulation tissue, and bone fragments) was delivered for microbiology and histology.

2.2. Determination of the Levels of Serum and Synovial Fluid PCT. PCT levels were quantified using a standard quantitative PCT enzyme immunoassay kit, according to the manufacturers' instructions (Elecsys ${ }^{\circledR}$ BRAHMS PCT test, Roche Diagnostics Ltd., Switzerland), on the Roche Cobas e601 analyzer. The lower limit of detection was 0.02 ng/mL. The specimens, either blood or synovial fluid, were collected and kept at room temperature $\left(10^{\circ} \mathrm{C}-25^{\circ} \mathrm{C}\right)$ and were measured within 2 hours. When synovial fluid cannot be measured within 2 hours, the specimen must be kept at approximately $2^{\circ} \mathrm{C}-8^{\circ} \mathrm{C}$ and must be measured within 24 hours. Due to the high viscosity of synovial fluid, the specimen was diluted at a ratio of $1: 4(100 \mu \mathrm{L}$ of synovial fluid sample with $300 \mu \mathrm{L}$ normal saline). Therefore, the synovial PCT level was then calculated from the measured PCT value multiplied by 4 , such as $0.08,0.12$, and $0.16 \mathrm{ng} / \mathrm{mL}$.

2.3. Statistical Analysis. Statistical analysis was carried out with MedCalc Statistical Software version 15.8 (MedCalc Software bvbv, Ostend, Belgium). Normally distributed continuous data were shown as mean \pm standard deviation (SD) and 
TABLE 3: Patient characteristics data.

\begin{tabular}{|c|c|c|c|c|}
\hline & $\begin{array}{l}\text { Total } \\
(\mathrm{n}=32)\end{array}$ & $\begin{array}{c}\text { PJI } \\
(n=20)\end{array}$ & $\begin{array}{c}\text { Aseptic loosening } \\
(n=12)\end{array}$ & p value \\
\hline Age, year $\diamond$ & $68(65-74)$ & $70(66-76)$ & $67 \pm 5$ & 0.098 \\
\hline Female gender $\odot$ & $25(78 \%)$ & $13(65 \%)$ & $12(100 \%)$ & $0.029^{*}$ \\
\hline BMI, $\mathrm{kg} / \mathrm{m}^{2} \triangle$ & $26.9 \pm 4.0$ & $26.3 \pm 4.5$ & $28.1 \pm 3.0$ & 0.229 \\
\hline Hip : Knee & $5: 27$ & $0: 20$ & $5: 7$ & $0.004^{*}$ \\
\hline Left : Right & $14: 18$ & $10: 10$ & $4: 8$ & 0.471 \\
\hline $\mathrm{CCI} \bullet$ & $3(2-4)$ & $3(3-4)$ & $2.5 \pm 1.0$ & 0.083 \\
\hline Systemic inflammatory disease@ & $2(6 \%)$ & $1(5 \%)$ & $1(8 \%)$ & 1.000 \\
\hline Receiving immunomodulating drugs@ & $2(6 \%)$ & $1(5 \%)$ & $1(8 \%)$ & 1.000 \\
\hline Body temperature $\left({ }^{\circ} \mathrm{C}\right)$ & $36.8(36.5-37.3)$ & $37.4 \pm 0.9$ & $36.6 \pm 0.2$ & $0.010^{*}$ \\
\hline WBC count $\left(\right.$ cell $\left./ \mathrm{mm}^{3}\right)$ & $6,620(5,755-10,250)$ & $9,170(1,700-28,100)$ & $6106 \pm 1433$ & $0.021^{*}$ \\
\hline
\end{tabular}

PJI: periprosthetic joint infection, BMI: body mass index, CCI: Charlson comorbidity index, and WBC: white blood cell; $\bullet$ : value presented as mean \pm SD or mean (IQR) and calculated with Mann-Whitney U test; $\odot$ : value presented as number of cases (percentage) and calculated with Fisher exact test or Chi-square test as appropriate; $\triangle$ : value presented with mean \pm standard deviation and calculated with unpaired $t$-test; value presented as the proportion of cases with that condition and calculated with Fisher exact test; ${ }^{*}$ significant $\mathrm{p}$ value with $\mathrm{p}<0.05$.

compared using student's $t$-test. Non-normally distributed continuous data were shown as median (interquartile range [IQR]) and compared using the Mann-Whitney U test. The categorical variables were presented as number of cases with proportion and compared with Chi-square or Fisher's exact test. A $p$ value $<0.05$ was considered statistically significant. Diagnostic accuracy of serum PCT and SF-PCT was assessed with receiver-operating characteristic (ROC) curves between the PJI and aseptic loosening groups. Sensitivity, specificity, positive likelihood ratio (LR+), negative likelihood ratio (LR), area under the ROC curve (AUC), and their 95\% confidence interval (CI) for any cut-off level were calculated.

\section{Results}

A total of 32 patients (5 revision hip arthroplasties and 27 revision knee arthroplasties) were recruited into our prospective study between 2015 and 2017. Regarding the International Consensus Criteria on PJI [17], 20 patients (20 revision knee arthroplasties) were classified in the PJI group and 12 patients ( 5 revision hip arthroplasties and 7 revision knee arthroplasties) were classified in the aseptic group. The patient characteristics data are presented in Table 3 . There were 7 males (22\%) and 25 females (78\%). The median patient age was 68 years (range $38-87$ years). The mean BMI was 26.9 $\pm 4.0 \mathrm{~kg} / \mathrm{m}^{2}$, and the median CCI was 3 (range $0-9$ ). Of these, 2 patients had preexisting rheumatoid arthritis (1 patient in each group) and were receiving immunomodulating drugs. No significant difference existed in age, BMI, operated side, $\mathrm{CCI}$, presence of systemic inflammatory disease, and concomitant immunomodulation drugs between both groups. However, the PJI group was significantly higher for male gender, revision knee arthroplasties, body temperature, and serum WBC count than the aseptic group ( $p<0.05$ all).

Tables 4 and 5 demonstrate the relevant clinical and laboratory findings according to the PJI definition [17] in both groups and the microbiological findings in our study. Of the 20 patients with PJI, 13 (65\%) had positive synovial fluid culture and $14(70 \%)$ had positive tissue culture. The

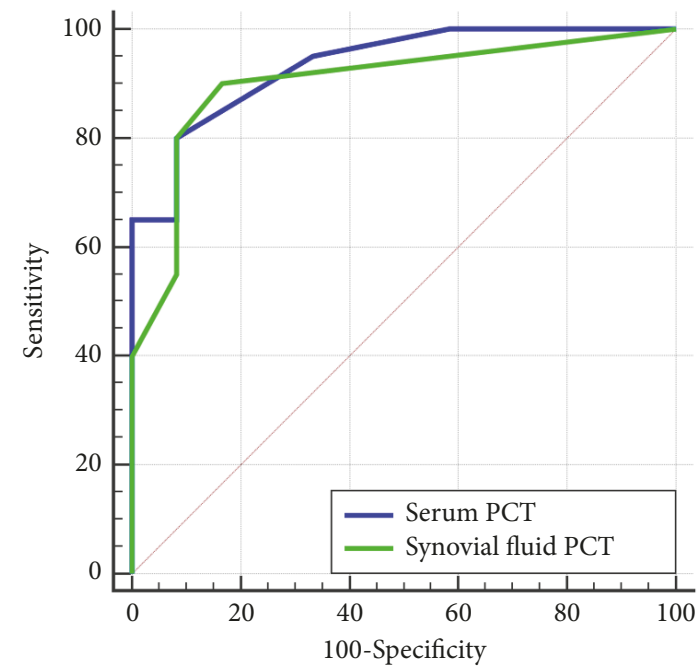

FIgURE 1: Receiver-operating characteristic (ROC) curves comparing ability between serum and synovial fluid PCT to detect periprosthetic joint infection (PJI) before revision arthroplasty.

most common microorganism from cultures-positive PJI was Streptococci $(\mathrm{n}=7,50 \%)$. The PJI group demonstrated significantly greater values in serum and synovial fluid markers related to infection than the aseptic loosening group $(p<$ 0.001 , all). The median serum PCT level (interquartile range: IQR) in the PJI and aseptic groups was 0.33 (0.08 to 2.79 ) and $0.04(0.03$ to 0.06$)$, respectively $(p<0.001)$. The median SFPCT (IQR) in the PJI and aseptic groups was 0.16 (0.12 to 0.26$)$ and 0.00 (0.00 to 0.00$)$, respectively $(p<0.001)$. Regarding the aseptic loosening group, the median serum PCT and SFPCT values from revision hip arthroplasties $(0.04$ and 0.00 $\mathrm{ng} / \mathrm{mL}$ ) did not significantly differ compared to those from revision knee arthroplasties (0.04 and $0.00 \mathrm{ng} / \mathrm{mL})(p=$ 0.400 and 0.287 , respectively).

Table 6 and Figure 1 show the diagnostic accuracy of PJI diagnosis by using serum PCT or SF-PCT in each cut-off 
TABLE 4: Relevant clinical and laboratory findings for PJI definition.

\begin{tabular}{|c|c|c|c|}
\hline & PJI $(n=20)$ & Aseptic loosening $(n=12)$ & p value \\
\hline Sinus tract presence $\odot$ & $0(0 \%)$ & $0(0 \%)$ & 1.00 \\
\hline Synovial fluid culture $\odot$ & $13(65 \%)$ & $0(0 \%)$ & $<0.001$ \\
\hline Tissue culture@ & $14(70 \%)$ & $0(0 \%)$ & $<0.001$ \\
\hline \multicolumn{4}{|l|}{ Serum markers } \\
\hline ESR, mm/hour $\triangle \nabla$ & $81 \pm 27$ & $18.5(12.5-36.5)$ & $<0.001$ \\
\hline CRP, mg/dL $\triangle$ & $149 \pm 98$ & $2.9 \pm 2.5$ & $<0.001$ \\
\hline Serum PCT, ng/mL $\nabla$ & $0.33(0.08-2.79)$ & $0.04(0.03-0.06)$ & $<0.001$ \\
\hline Hip & $\mathrm{n} / \mathrm{a}$ & $0.04(0.02-0.06)$ & $\mathrm{n} / \mathrm{a}$ \\
\hline Knee & $0.33(0.08-2.79)$ & $0.04 \pm 0.04$ & 0.001 \\
\hline \multicolumn{4}{|l|}{ Synovial fluid markers } \\
\hline Synovial fluid WBC, cell $/ \mathrm{mm}^{3} \nabla \triangle$ & $78,920(3,420-335,400)$ & $1350 \pm 827$ & $<0.001$ \\
\hline \%Neutrophil $\triangle$ & $90.8 \pm 6.2$ & $54.9 \pm 17.2$ & $<0.001$ \\
\hline SF-PCT, ng/mL $\nabla$ & $0.16(0.12-0.26)$ & $0.00(0.00-0.00)$ & $<0.001$ \\
\hline Hip & $\mathrm{n} / \mathrm{a}$ & 0.000 .00 & $\mathrm{n} / \mathrm{a}$ \\
\hline Knee & $0.16(0.12-0.26)$ & $0.00(0.00-0.06)$ & 0.004 \\
\hline
\end{tabular}

○: value presented as number of cases (percentage), $\triangle$ : value presented as mean \pm standard deviation, $\nabla$ : value presented as median (interquartile range), and n/a: not available.

TABLE 5: Microbiological findings of culture-diagnosed PJI among 14 episodes.

\begin{tabular}{lc}
\hline Micro-organisms & No. (\%) \\
\hline Gram positive & $1(7 \%)$ \\
Staphylococcus aureus & $1(7 \%)$ \\
CNS & $7(50)$ \\
Streptococci & \\
Gram positive & $3(21 \%)$ \\
Escherichia coli & $1(7 \%)$ \\
Proteus mirabilis & $1(7 \%)$ \\
Other &
\end{tabular}

CNS: coagulative negative staphylococcus; ${ }^{\text {a }}$ Propionibacterium spp.

value and ROC curve comparison between serum PCT and SF-PCT. The cut-off references of serum PCT were set as $0.1,0.3$, and $0.5 \mathrm{ng} / \mathrm{mL}$, whereas those of SF-PCT were set as $0.08,0.12$, and $0.16 \mathrm{ng} / \mathrm{mL}$. Regarding the accuracy of the serum PCT test with the standard cut-off reference level as 0.5 $\mathrm{ng} / \mathrm{mL}$, the sensitivity, specificity, LR+, and LR- were $40.0 \%$, $100.0 \%$, not available, and 0.60 , respectively. However, with the lower cut-off level as $0.1 \mathrm{ng} / \mathrm{mL}$, the serum PCT test showed sensitivity, specificity, LR+, and LR- as $65.0 \%, 91.7 \%$, 7.80 , and 0.38 , respectively. The AUC of 0.5 and $0.1 \mathrm{ng} / \mathrm{mL}$ cut-off levels was 0.70 and 0.78 , respectively.

Regarding the accuracy of the SF-PCT test for PJI diagnosis, the cut-off value as $0.08 \mathrm{ng} / \mathrm{mL}$ resulted in sensitivity of $90.0 \%$, specificity of $83.3 \%$, LR+ of 5.40 , and LR- of 0.12 . Conversely, the higher cut-off level as $0.12 \mathrm{ng} / \mathrm{mL}$ showed sensitivity of $80.0 \%$, specificity of $91.7 \%$, LR+ of 9.40 , and LRof 0.22 . The AUC of 0.08 and $0.12 \mathrm{ng} / \mathrm{mL}$ cut-off levels was 0.87 and 0.86 , respectively (Table 6 ).

\section{Discussion}

Periprosthetic joint infection (PJI) is one of the most severe and costly complications following total joint arthroplasty. Although there is an international consensus for the definition of PJI, no single "gold standard" test currently exists for diagnosing PJI. Recently, many studies have reported the usefulness of synovial fluid cytokines-such as interleukin6 , c-reactive protein, and alpha-defensin-as alternative and better diagnostic markers for PJI compared to the standard technique $[7,8,18-21]$. The overall sensitivity and specificity of these markers were more than $80 \%$ and $90 \%$, respectively [7]. However, to our knowledge, although some biomarkers have demonstrated excellent diagnostic performance for PJI, the comparison of diagnostic accuracy between these biomarkers did not achieve statistical significance [8]. Moreover, according to the current evidence on these new biomarkers, serum PCT is a promising and reliable test, but the utility of synovial fluid PCT for detecting PJI has not been clearly demonstrated.

The results of this study show that both serum PCT and SF-PCT could be used as diagnostic biomarkers to support clinicians in differentiating PJI from aseptic loosening. The PJI group had significantly higher serum PCT and SF-PCT values, the same as serum ESR and CRP $(p<0.001$ all $)$, compared with the aseptic loosening group (Table 4). Using ROC curve analysis, the present study demonstrates that serum PCT, with the standard cut-off level as $0.5 \mathrm{ng} / \mathrm{mL}$ (a sensitivity of $40 \%$, a specificity of $100 \%$, and AUC of 0.70 ), is comparable to PCT from the previous meta-analysis (pooled sensitivity of $53 \%$, pooled specificity of $92 \%$, and AUC of 0.76 ) [15]. Additionally, this study also reveals that, with the lower serum PCT cut-off level as $0.1 \mathrm{ng} / \mathrm{mL}$, the diagnostic accuracy of serum PCT could be further improved to sensitivity of $65 \%$, persistently good specificity (92\%), and AUC of 0.78 
TABLE 6: Diagnostic accuracy of PJI diagnosis using serum or synovial fluid procalcitonin.

\begin{tabular}{|c|c|c|c|c|c|}
\hline & Sensitivity & Specificity & AUC & LR+ & LR- \\
\hline \multicolumn{6}{|c|}{ Serum PCT (ng/mL) } \\
\hline 0.1 & $65.0(40.8-84.6)$ & $91.7(61.5-99.8)$ & $0.78(0.60-0.91)$ & $7.80(1.16-52.35)$ & $0.38(0.21-0.71)$ \\
\hline 0.3 & $50.0(27.2-72.8)$ & $100.0(73.5-100.0)$ & $0.75(0.57-0.89)$ & $\mathrm{n} / \mathrm{a}$ & $0.47(0.29-0.76)$ \\
\hline 0.5 & $40.0(19.1-64.0)$ & $100.0(73.5-100.0)$ & $0.70(0.51-0.85)$ & $\mathrm{n} / \mathrm{a}$ & $0.60(0.42-0.86)$ \\
\hline \multicolumn{6}{|c|}{ Synovial fluid PCT (ng/mL) } \\
\hline 0.08 & $90.0(68.3-98.8)$ & $83.3(51.6-97.9)$ & $0.87(0.70-0.96)$ & $5.40(1.51-19.30)$ & $0.12(0.03-0.46)$ \\
\hline 0.12 & $80.0(56.3-94.3)$ & $91.7(61.5-99.8)$ & $0.86(0.69-0.96)$ & $9.60(1.45-63.50)$ & $0.22(0.09-0.53)$ \\
\hline 0.16 & $55.0(31.5-76.9)$ & $91.7(61.5-99.8)$ & $0.73(0.55-0.87)$ & $6.60(0.97-44.93)$ & $0.49(0.29-0.82)$ \\
\hline
\end{tabular}

AUC: area under curve, LR+: positive likelihood ratio, LR-: negative likelihood ratio, and PCT: procalcitonin.

(Table 5). However, serum PCT with a lower cut-off level should be used with caution and may need future larger studies to ensure an effective strategy implementation.

Regarding ROC curve analysis, SF-PCT showed an ability to be a more valuable biomarker for identifying PJI from aseptic loosening than serum PCT. With a cut-off level as 0.08 $\mathrm{ng} / \mathrm{mL}$, SF-PCT showed the greatest accuracy with sensitivity of $90 \%$, specificity of $83 \%$, LR+ of 5.40 , LR- of 0.12 , and AUC of 0.87 (Table 5). These high sensitivity, high LR+, and very low LR- characteristics are all good indicators for ruling in and out the diagnosis of PJI [22], especially for preoperative and intraoperative settings. This is because the PCT test would take only 30 minutes to perform in the laboratory and may have the potential to become a point-of-care test in the patients who obtain the synovial fluid. Additionally, this study also found that, according to the PJI group, the concentration of PCT in blood (median value $0.33 \mathrm{ng} / \mathrm{mL}$, interquartile range $0.08 \mathrm{ng} / \mathrm{mL}-2.79 \mathrm{ng} / \mathrm{mL}$ ) seemed to be greater (about two times) than those in joint fluid (median value $0.16 \mathrm{ng} / \mathrm{mL}$, interquartile range $0.12 \mathrm{ng} / \mathrm{mL}-0.26 \mathrm{ng} / \mathrm{mL}$ ). However, the difference did not reach statistical significance $(p=0.20)$ (Table 4). This could imply that the cut-off reference for SFPCT should be different from those for serum PCT, the same as the other synovial fluid biomarker [21].

Concerning the comparison of the diagnostic performance between synovial fluid biomarkers for PJI diagnosis, although this study demonstrated a good accuracy of SF-PCT for PJI diagnosis with an AUC of 0.87, this diagnostic accuracy appeared to be slightly inferior to biomarkers from previous studies-such as CRP, IL-6, and alpha-defensin - with AUC between 0.90 and 0.99 [18, 20, 23, 24]. However, due to the previously noted potential of the PCT test, we still recommend using SF-PCT as a complementary tool with the standard technique for diagnosing PJI.

This study also had some limitations. Firstly, due to the prospective cohort nature in only one university hospital center, our sample size was relatively small and included both knee and hip patients. Therefore, future longitudinal studies with a larger sample size and a specific analytic approach for revision knee or hip arthroplasties would require determining the usefulness of SF-PCT for detecting PJI. Secondly, this study did not include patients with prior antibiotics therapy or with concomitant disease that might affect SF-PCT, such as crystal-induced arthritis or malignancy [13]. Lastly, a diagnostic accuracy comparison between SF-PCT and other biomarkers was not performed. However, the information related to other biomarkers is already published.

\section{Conclusion}

The accuracy of SF-PCT was significantly higher than that of serum PCT. Therefore, SF-PCT may be used as an alternative indicator in the differential diagnosis of PJI from aseptic loosening in cases where patients are undergoing revision hip or knee arthroplasty. However, further prospective studies with a larger sample size are required to validate the usefulness of SF-PCT.

\section{Data Availability}

The data used to support the findings of this study are available from the corresponding author upon request.

\section{Conflicts of Interest}

All of the authors declare that they have no conflicts of interest.

\section{Authors' Contributions}

Paphon Sa-ngasoongsong and Siwadol Wongsak were main researchers who designed and performed this study and prepared the manuscript. Chavarat Jarungvittayakon, Kawee Limsamutpetch, and Thanaphot Channoom were orthopaedic trauma surgeons who assisted in data collection and manuscript preparation. Viroj Kawinwonggowit was senior orthopaedic consultant who assisted in research process.

\section{Acknowledgments}

The authors wish to thank Department of Orthopedics, Faculty of Medicine Ramathibodi Hospital, Mahidol University, for all of the kind help and permission to carry out this study. This study was funded by Faculty of Medicine Ramathibodi hospital, Mahidol University. 


\section{References}

[1] K. J. Bozic, S. M. Kurtz, E. Lau et al., "The epidemiology of revision total knee arthroplasty in the united states," Clinical Orthopaedics and Related Research, vol. 468, no. 1, pp. 45-51, 2010.

[2] K. J. Bozic, S. M. Kurtz, E. Lau, K. Ong, D. T. P. Vail, and D. J. Berry, "The epidemiology of revision total hip arthroplasty in the united states," The Journal of Bone \& Joint Surgery, vol. 91, no. 1, pp. 128-133, 2009.

[3] J. S. Coste, S. Reig, C. Trojani, M. Berg, G. Walch, and P. Boileau, "The management of infection in arthroplasty of the shoulder," The Journal of Bone \& Joint Surgery (British Volume), vol. 86-B, no. 1, pp. 65-69, 2004.

[4] K. Yuan, W.-D. Li, Y. Qiang, and Z.-M. Cui, "Comparison of procalcitonin and C-reactive protein for the diagnosis of periprosthetic joint infection before revision total hip arthroplasty," Surgical Infections, vol. 16, no. 2, pp. 146-150, 2015.

[5] C. Deirmengian, K. Kardos, P. Kilmartin et al., "The Alphadefensin Test for Periprosthetic Joint Infection Outperforms the Leukocyte Esterase Test Strip," Clinical Orthopaedics and Related Research, vol. 473, no. 1, pp. 198-203, 2015.

[6] A. Elgeidi, A. E. Elganainy, N. Abou Elkhier, and S. Rakha, "Interleukin-6 and other inflammatory markers in diagnosis of periprosthetic joint infection," International Orthopaedics, vol. 38, no. 12, pp. 2591-2595, 2014.

[7] Y. S. Lee, K. Koo, H. J. Kim et al., "Synovial Fluid Biomarkers for the Diagnosis of Periprosthetic Joint Infection," The Journal of Bone \& Joint Surgery, vol. 99, no. 24, pp. 2077-2084, 2017.

[8] A. Saleh, D. Ramanathan, M. B. P. Siqueira, A. K. Klika, W. K. Barsoum, and C. A. H. Rueda, "The diagnostic utility of synovial fluid markers in periprosthetic joint infection: A systematic review and meta-analysis," Journal of the American Academy of OrthopaedicSurgeons, vol. 25, no. 11, pp. 763-772, 2017.

[9] N. G. Morgenthaler, J. Struck, C. Fischer-Schulz, E. SeidelMueller, W. Beier, and A. Bergmann, "Detection of procalcitonin (PCT) in healthy controls and patients with local infection by a sensitive ILMA," Clinical Laboratory, vol. 48, no. 5-6, pp. 263-270, 2002.

[10] F. Ferriere, "Procalcitonin, a new marker for bacterial infections," Annales de Biologie Clinique, vol. 58, no. 1, pp. 49-59, 2000.

[11] A. Abd El-Azim, M. Sultan, U. Alkholi, and N. Abd Al-monem, "Serum procalcitonin in viral and bacterial meningitis," Journal of Global Infectious Diseases, vol. 3, no. 1, pp. 14-18, 2011.

[12] K. Maharajan, D. K. Patro, J. Menon et al., "Serum Procalcitonin is a sensitive and specific marker in the diagnosis of septic arthritis and acute osteomyelitis," Journal of Orthopaedic Surgery and Research, vol. 8, no. 1, article no. 19, 2013.

[13] K. Saeed, M. Dryden, A. Sitjar, and G. White, "Measuring synovial fluid procalcitonin levels in distinguishing cases of septic arthritis, including prosthetic joints, from other causes of arthritis and aseptic loosening," Infection, vol. 41, no. 4, pp. 845-849, 2013.

[14] C. Wang, D. Zhong, Q. Liao, L. Kong, A. Liu, and H. Xiao, "Procalcitonin levels in fresh serum and fresh synovial fluid for the differential diagnosis of knee septic arthritis from rheumatoid arthritis, osteoarthritis and gouty arthritis," Experimental and Therapeutic Medicine, vol. 8, no. 4, pp. 1075-1080, 2014.

[15] K. Xie, X. Qu, and M. Yan, "Procalcitonin and $\alpha$-Defensin for Diagnosis of Periprosthetic Joint Infections," The Journal of Arthroplasty, vol. 32, no. 4, pp. 1387-1394, 2017.
[16] S. Ali, A. Christie, and A. Chapel, "The pattern of procalcitonin in primary total hip and knee arthroplasty and its implication in periprosthetic infection," Journal of Clinical Medicine Research, vol. 1, no. 2, pp. 90-94, 2009.

[17] J. Parvizi and T. Gehrke, "Definition of periprosthetic joint infection," The Journal of Arthroplasty, vol. 29, no. 7, p. 1331, 2014.

[18] K. Xie, K. Dai, X. Qu, and M. Yan, "Serum and Synovial Fluid Interleukin-6 for the Diagnosis of Periprosthetic Joint Infection," Scientific Reports, vol. 7, no. 1, 2017.

[19] J. Pupaibool, E. J. Fulnecky, R. L. Swords, W. W. Sistrunk, and A. D. Haddow, "Alpha-defensin-novel synovial fluid biomarker for the diagnosis of periprosthetic joint infection," International Orthopaedics, vol. 40, no. 12, pp. 2447-2452, 2016.

[20] M. W. Tetreault, N. G. Wetters, M. Moric, C. E. Gross, and C. J. Della Valle, "Is Synovial C-reactive Protein a Useful Marker for Periprosthetic Joint Infection?" Clinical Orthopaedics and Related Research, vol. 472, no. 12, pp. 3997-4003, 2014.

[21] T. M. Randau, M. J. Friedrich, B. Wimmer et al., "Interleukin6 in serum and in synovial fluid enhances the differentiation between periprosthetic joint infection and aseptic loosening," PLoS ONE, vol. 9, no. 2, Article ID e89045, 2014.

[22] A. M. Simundic, "Measures of Diagnostic Accuracy: Basic Definitions," EJIFCC, vol. 19, no. 4, pp. 203-211, 2009.

[23] T. Bonanzinga, A. Zahar, M. Dütsch, C. Lausmann, D. Kendoff, and T. Gehrke, "How Reliable Is the Alpha-defensin Immunoassay Test for Diagnosing Periprosthetic Joint Infection? A Prospective Study," Clinical Orthopaedics and Related Research, vol. 475, no. 2, pp. 408-415, 2017.

[24] M. Wyatt, A. Beswick, S. Kunutsor, M. Wilson, M. Whitehouse, and A. Blom, "The Alpha-Defensin Immunoassay and Leukocyte Esterase Colorimetric Strip Test for the Diagnosis of Periprosthetic Infection," The Journal of Bone \& Joint Surgery, vol. 98, no. 12, pp. 992-1000, 2016. 


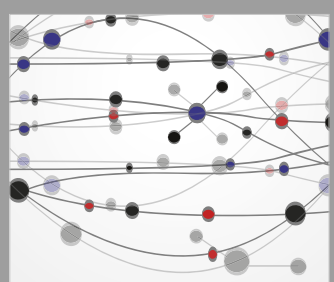

The Scientific World Journal
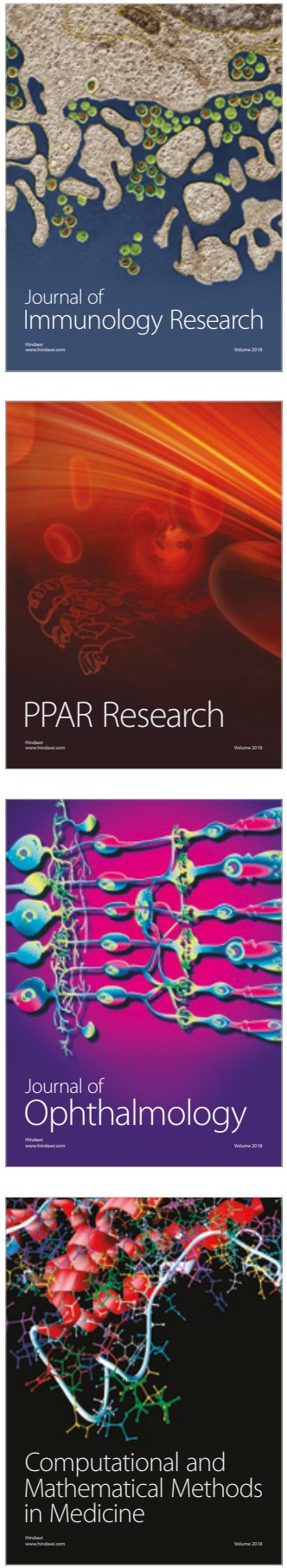

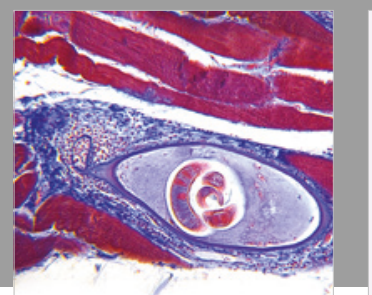

Gastroenterology Research and Practice

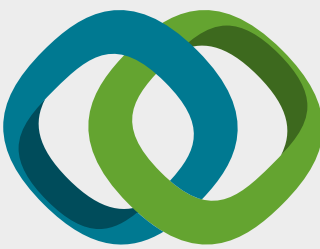

\section{Hindawi}

Submit your manuscripts at

www.hindawi.com
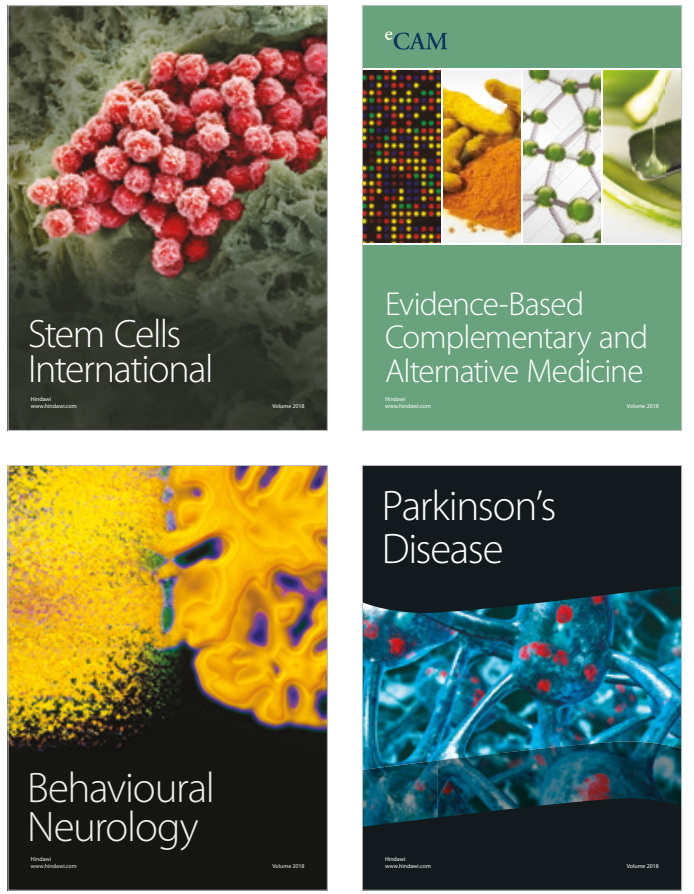

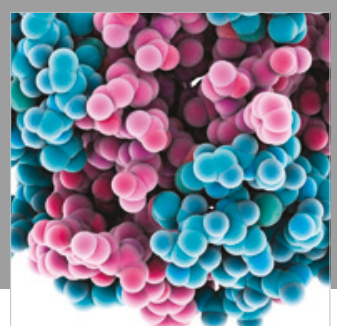

ournal of

Diabetes Research

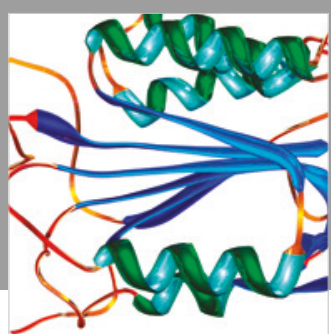

Disease Markers
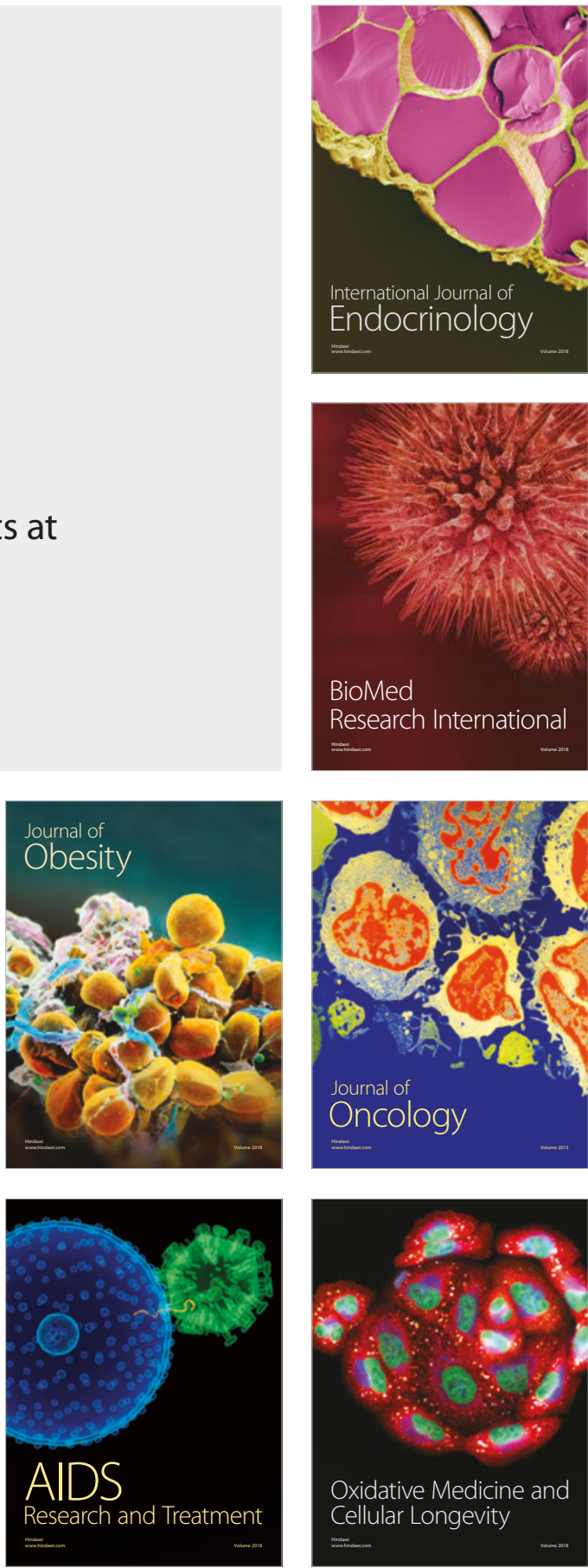